\section{Cartridge Methods for Oligonucleotide Purification}

This unit provides protocols for DMTr (trityl)-selective (see Basic Protocol 1) and affinity desalting (see Basic Protocol 2) purification of oligonucleotides. The protocols are applicable for many of the convenient, disposable products for rapid oligonucleotide purification, clean-up by selective adsorption, and elution on solid-phase media. Many of the products are prepackaged, one-time use cartridges or columns filled with affinity or size-exclusion media.

\section{DMTr-SELECTIVE CARTRIDGE PURIFICATION}

This protocol describes purification of tritylated oligonucleotides with the Oligonucleotide Purification Cartridge (OPC, PE Applied Biosystems). The OPC can desalt, detritylate, and purify tritylated oligonucleotides directly from the ammonium hydroxide solution after cleavage/deprotection (McBride et al., 1988; Applied Biosystems, 1991; Blocker et al., 1991).

The purity level achieved by OPC is appropriate for such common applications as sequencing primers, PCR primers, and hybridization probes. Upon loading, the tritylated oligonucleotide is retained by the hydrophobic polystyrene medium, while the salts, non-tritylated oligonucleotides, and other impurities are not retained, and are washed away. The trityl group of the OPC-bound oligonucleotide is removed with a brief acid treatment, and the full-length, detritylated oligonucleotide is eluted with $\sim 1 \mathrm{~mL}$ of a $20 \%$ acetonitrile solution.

\section{Materials}

$20 \%(\mathrm{v} / \mathrm{v})$ and $100 \%$ acetonitrile

$2 \mathrm{M}$ triethylammonium acetate (TEAA)

Tritylated oligonucleotide in ammonia deprotection solution

$3 \%(\mathrm{v} / \mathrm{v})$ trifluoroacetic acid (TFA)

1.5 $\mathrm{M}$ ammonium hydroxide

Oligonucleotide Purification Cartridges (OPC; PE Biosystems)

Vacuum manifold (Analytichem or National Scientific; optional) with an in-line trap for waste and a water aspirator pump or small vacuum pump

5-mL disposable plastic syringes with exposed luer tips

\section{Prepare the cartridge}

1. Mount the OPC with a clamp and insert an empty 5-mL syringe barrel on the top of the cartridge.

Multiple cartridges can optionally be used with a vacuum manifold system.

2. Fill the syringe barrel with $5 \mathrm{~mL}$ of $100 \%$ acetonitrile. Insert and depress the plunger to pass the reagent through the OPC (into an appropriate waste container) over an elapsed time of $\sim 15$ to $30 \mathrm{sec}$.

All reagents should be eluted from the OPC cartridge dropwise.

3. Repeat step 2 with $5 \mathrm{~mL}$ of $2 \mathrm{M}$ TEAA.

\section{Prepare the sample}

4. Dilute the oligonucleotide ammonia solution (up to 20 OD units) with an equal volume of water (e.g., normally $\sim 1 \mathrm{~mL}$ ).
UNIT 10.7

BASIC

PROTOCOL 1

Purification and Analysis of Synthetic Nucleic Acids and Components

\subsection{1}

Supplement 1 
5. Pass the diluted solution through the $\mathrm{OPC}$ at a rate of $\sim 1$ drop/sec. Collect the eluate and pass it through a second time.

6. Wash the $\mathrm{OPC}$ with $5 \mathrm{~mL}$ of $1.5 \mathrm{M}$ ammonium hydroxide, then with $10 \mathrm{~mL}$ deionized water, allowing the effluents to pass to waste.

7. Fill the syringe barrel with $5 \mathrm{~mL}$ of $3 \%$ trifluoroacetic acid (TFA) and pass $\sim 2 \mathrm{~mL}$ through the OPC to waste. Let stand for $5 \mathrm{~min}$ before passing the remainder through to waste.

8. Pass $10 \mathrm{~mL}$ of deionized water through the $\mathrm{OPC}$ to waste.

9. For oligonucleotides $>40 \mathrm{nt}$, pass $5 \mathrm{~mL}$ of $1.5 \mathrm{M}$ ammonium hydroxide through the column, to waste, then pass $5 \mathrm{~mL}$ of water through the OPC to waste. If oligonucleotide is $\leq 40 \mathrm{nt}$, skip to step 10 .

\section{Collect the purified oligonucleotide}

10. Elute the column, with $1 \mathrm{~mL}$ of $20 \%$ acetonitrile dropwise, and collect the purified oligonucleotide in a $1.5-\mathrm{mL}$ microcentrifuge tube. Quantify by OD measurement.

BASIC PROTOCOL 2
Cartridge Methods for Oligonucleotide Purification

\section{AFFINITY CARTRIDGE DESALTING OF DETRITYLATED OLIGONUCLEOTIDES}

This protocol details the use of an affinity cartridge to desalt and concentrate detritylated oligonucleotides, with the capacity to yield up to $50 \mathrm{OD}$ units of desalted oligonucleotide per cartridge. No purification is effected, beyond removal of species that fail to bind to the cartridge medium, which typically include salts, solvents, reagents, and very short failure oligonucleotides. The OPC is one example of several commercially available products that can render oligonucleotides free of salts, trace amounts of solvents, and other small molecules by immobilizing, washing, and eluting on an affinity medium. This desalting protocol is useful for (1) trityl-off oligonucleotides; (2) fluorescent dye-labeled, biotin-labeled, and other labeled oligonucleotides; (3) RNA oligonucleotides, after desilylation/deprotection; (4) polyacrylamide gel purification extracts; and (5) anion-exchange HPLC purification fractions. An alternative to this protocol is ethanol precipitation (UNIT 10.3).

\section{Materials}

$0.1 \mathrm{M}$ triethylammonium acetate (TEAA)

$50 \%(\mathrm{v} / \mathrm{v})$ acetonitrile

Additional reagents and equipment for cartridge purification (see Basic Protocol 1)

1. Mount cartridge and wash with acetonitrile and TEAA (see Basic Protocol 1, steps 1 to 3$)$.

2. Concentrate up to $50 \mathrm{OD}$ units of the oligonucleotide to dryness under vacuum.

For maximum binding capacity, all organic solvents and ammonium hydroxide must be removed.

3. Dissolve the completely dried oligonucleotide in $2 \mathrm{~mL}$ of $0.1 \mathrm{M}$ TEAA.

4. Pass the oligonucleotide solution through the OPC at a rate of $\sim 1 \mathrm{drop} / \mathrm{sec}$. Collect the eluate and pass it through a second time.

5. Pass $15 \mathrm{~mL}$ of $0.1 \mathrm{M}$ TEAA through the OPC to waste. 
6. Elute the column with $1 \mathrm{~mL}$ of $50 \%$ acetonitrile dropwise, and collect the desalted oligonucleotide in a $1.5-\mathrm{mL}$ microcentrifuge tube. Quantify by OD measurement.

\section{COMMENTARY}

\section{Background Information}

\section{DMTr-selective cartridge purification}

DMTr-selective cartridge purification separates oligonucleotides by the same hydrophobic interaction principle as reversed-phase HPLC (UNIT 10.5). Instead of a constant gradient elution with an organic modifier (i.e., acetonitrile) as in HPLC, the cartridge method is a batch-loading, washing, and elution process. The costs and complexity of the cartridge methods, in general, are far less than HPLC or PAGE (UNIT 10.4). However, the cartridge methods do not provide concomitant analytical information and are limited in capacity and efficiency of purification. Other cartridges, with similar protocols, are commercially available for purification and desalting of oligonucleotides. Check with the manufacturers for their optimum protocols.

The DMTr group at the $5^{\prime}$ terminus is very hydrophobic and allows for specific binding of the tritylated oligonucleotide with the stationary, hydrophobic polystyrene adsorbent of OPC. Therefore, the product can be separated from other components of the crude synthesis mixture which do not bind. The crude tritylated oligonucleotide is conveniently loaded from the concentrated ammonium hydroxide cleavage/deprotection solution onto the OPC. Other cartridges packed with reversed-phase silica adsorbent, are not compatible with the low- and high-pH reagents used in Basic Protocol 1.

\section{Affinity desalting}

The affinity desalting protocol (Basic Protocol 2) takes advantage of the hydrophobic interactions of even untritylated oligonucleotides with the cartridge adsorbent. In the absence of the denaturing solution, ammonium hydroxide, which limits binding, the desalting protocol can bind a relatively large quantity of oligonucleotide and remove large quantities of impurities. Desalting removes impurities, such as deprotection byproducts, which may hinder some applications. Also, removal of UV-absorbing small molecules results in more accurate UV quantitation. Basic Protocol 2 is particularly useful to remove the large quantities of salts from anion-exchange HPLC purification fractions (UNIT 10.5) and the buffer and gel debris from PAGE purified oligonucleotides (UNIT 10.4).
Many labeled oligonucleotides can be partially separated from unlabeled oligonucleotides by affinity cartridges (Basic Protocol 2). Fluorescent dyes, biotin, and many other labels impart added hydrophobicity to confer reasonable binding capacity. When attempting to separate labeled oligonucleotides from unlabled oligonucleotides, it may be necessary to replace step 5 with $5 \mathrm{~mL}$ of $3 \%$ to $12 \%$ acetonitrile in $0.1 \mathrm{M}$ TEAA. HPLC can be used to help determine the best conditions for a particular label.

Many cartridge purifications can be conducted in parallel with a simple, commercially available vacuum manifold, e.g., Vac Elut SPS 24 , or Vac Elut 10-place manifold (Analytichem) with a $12 \times 75$-mm test tube rack. The 12-position manifold (National Scientific) is also suitable. In addition to vastly increased throughput, manifolds require fewer manual manipulations. The cartridges are mounted to the suction element of the manifold and the syringe barrels are merely filled with reagents, remaining connected to the cartridge at all times. Many commercial units give excellent results, with no cross-contamination of samples (Applied Biosystems, 1991).

\section{Size-exclusion filtration}

Oligonucleotides can be separated from smaller molecules by passage through a medium, such as Sephadex, which allows separation based on molecular weight (this protocol is not provided here). Large molecules follow a shorter mean path length, due to exclusion from the particle pores, than smaller molecules. Unlike hydrophobic media in affinity methods, size-exclusion media have little or no hydrophobic or ion-exchange interactions with oligonucleotides. Size-exclusion filtration, also called gel-filtration or gel-permeation chromatography, is rapid and convenient but has limited separating power across a narrow mass range. The Sephadex medium is available as dry powder to be preswollen in aqueous solution, or prepacked in columns. The MicroSpin and NAP columns (Pharmacia Biotech) have quick protocols and require minimal labor. Sample volumes of 100 to $150 \mu \mathrm{L}$ are optimally processed in a MicroSpin S-200 HR column in a microcentrifuge, whereas sample volumes between 0.2 and $2.5 \mathrm{~mL}$ require the appropri-
Purification and Analysis of Synthetic Nucleic Acids and Components 
ately scaled NAP column under gravity flow. The medium is equilibrated in the solution of choice, such as water. The oligonucleotide should be loaded in a minimum volume, such as $200 \mu \mathrm{L}$ water, and all eluate should be collected. The oligonucleotide will elute in the void volume, while smaller species will be slightly retained.

A related technique utilizes disposable, membrane-based, ultrafiltration devices, e.g., Microcon (Amicon), that operate in a microcentrifuge. In this method, the "large" oligonucleotides (and other, higher-molecular-weight nucleic acids) are retained above the membrane, whereas the "small" impurities flow through and are discarded. In effect, the oligonucleotide is continuously concentrated in a diminishing volume, which is an added benefit. Disposable devices are available that can accommodate a variety of different volumes and mass ranges.

\section{Critical Parameters and Troubleshooting}

The efficiency of DMTr-selective cartridge purification (Basic Protocol 1) depends largely on the efficiency of the synthesis process. When the detritylation, capping, and oxidation steps during synthesis are efficient, only the correctsequence oligonucleotide should bear the DMTr group, and a DMTr-selective purification protocol can furnish a very pure product. Moderately low coupling efficiency (95\% to 97\% average yield per cycle) will not limit purification as long as these other steps in the synthesis process are quantitative. Other side reactions, such as depurination and extraneous growth off the synthesis support, lead to trityl heterogeneity and limit purification. Shorter tritylated oligonucleotides have higher affinity for the OPC than longer tritylated oligonucleotides because they are more hydrophobic and tend to be more pure than long oligonucleotides. For example, a 20-nt oligonucleotide may yield 5 OD units, whereas a 100-nt oligonucleotide may yield only 1 OD unit of purified product. Normally, when oligonucleotides are purified (Basic Protocol 1) soon after synthesis, e.g., within a few days, the trityl group is stable in the ammonium hydroxide solution. However, upon prolonged storage, even in a freezer, the trityl group will cleave regardless of the solution it is stored in. Therefore, DMTr-selective purifications (see Basic Protocol 1; also see UNIT 10.5 for reversed-phase HPLC) should be conducted within a few days after synthesis. Triethylammonium is the re- sulting counterion of oligonucleotides purified by Basic Protocols 1 and 2. If it is necessary to exchange the triethylammonium counterion with sodium, the oligonucleotide can be precipitated in ethanol and sodium acetate (UNIT 10.5) after cartridge purification.

In Basic Protocols 1 and 2, the initial acetonitrile wash wets and swells the adsorbent. The triethylammonium acetate wash removes the acetonitrile and provides triethylammonium counter-ions to help bind the oligonucleotide. The presence of ammonia in the loading solution of the oligonucleotide provides the desired denaturing medium but limits the binding capacity of tritylated oligonucleotides. If ammonia is partially removed (by leaving for several minutes in a Speedvac evaporator or letting the sample remain uncapped for $15 \mathrm{~min}$ ), the binding capacity of DMTr oligonucleotides will increase to yield $\sim 10$ OD units of purified, detritylated oligonucleotide. A dilute ammonia wash follows loading to insure that non-DMTr oligonucleotides have been removed. The water wash removes residual ammonia so it will not neutralize the TFA (trifluoroacetic acid) in the following step. Detritylation with $5 \mathrm{~mL}$ of $3 \%$ TFA requires $5 \mathrm{~min}$. The detritylation can sometimes be observed as a slight orange or pink blush to the support. Even though it no longer has a trityl group, at neutral and low $\mathrm{pH}$, the purified oligonucleotide is retained while washing with water to remove residual acidic TFA.

For oligonucleotides $>40 \mathrm{nt}$, the second dilute ammonia wash serves to remove shorter oligonucleotides while allowing longer oligonucleotide to be retained. A water wash follows to remove residual ammonium hydroxide. Elution of the purified oligonucleotide occurs with $20 \%$ acetonitrile. The cleaved dimethoxytrityl alcohol remains on the OPC and does not contaminate the purified oligonucleotide.

Reuse of any purification matrix can lead to problems with carryover and sample contamination. Avoiding these problems is especially important in synthetic DNA applications because of the potential for errant priming and hybridization. Analytical HPLC studies demonstrate that $-5 \%$ of an oligonucleotide product is carried over as a contaminant into a second, different oligonucleotide sequence upon reuse of a purification cartridge. Also, the DMTr that is released upon TFA treatment is very tightly bound, resulting in diminished capacity and selectivity for DMTr oligonucleotides. The saf- 
est, most reliable solution is a disposable onetime use format.

For longer-term storage (i.e., >6 months), oligonucleotides that have been purified by OPC (see Basic Protocol 1) should be stored as dry solids at $-15^{\circ} \mathrm{C}$.

\section{Anticipated Results}

In Basic Protocol 1, 10 to 20 OD units of a crude, tritylated oligonucleotide (i.e., an entire $40 \mathrm{nmol}$ scale and most, or all of a $0.2 \mu \mathrm{mol}$ scale synthesis) can be loaded, to yield 1 to 10 OD units of purified product. The yield depends on initial purity, length, and concentration of ammonium hydroxide in the loading solution. Yield is measured in OD units by UV absorbance at $260 \mathrm{~nm}$, converted to mass by using the approximation of $33 \mu \mathrm{g}$ per OD unit. Oligonucleotides purified by Basic Protocol 1 are typically $>90 \%$ pure by integrated peak area HPLC analysis, which compares well with HPLC (UNIT 10.5) and PAGE (UNIT 10.4) methods. In Basic Protocol 2, the yield can be as high as 50 OD units of desalted product.

Synthetic oligoribonucleotides (RNA) can also be desalted and purified by DMTr-selective, affinity cartridges (Mullah and Andrus, 1996). Purification of phosphorothioate oligonucleotide analogs by the cartridge method is also very efficient. Since phosphorothioates are more hydrophobic, use $1 \mathrm{~mL}$ of $35 \%$ acetonitrile to elute them (Basic Protocol 1, step 10).

\section{Time Considerations}

Basic Protocol 1 requires $\sim 15$ to $20 \mathrm{~min}$. Basic Protocol 2 requires $\sim 10$ to $15 \mathrm{~min}$. Use of a vacuum manifold will speed up purification of even a single oligonucleotide and results in large time savings when purifying a larger number. For example, 10 oligonucleotides can be purified by Basic Protocol 1 on a vacuum manifold in $<30 \mathrm{~min}$.

\section{Literature Cited}

Applied Biosystems. 1991. New Applications for the Oligonucleotide Purification Cartridge. User Bulletin No. 59.

Blocker, H., Frank, R., Heisterberg-Moutsis, G., Kurth, G., and Meyerhans, A. 1991. Improved Process for the Purification of Synthetic Oligonucleotides. U.S. Patent No. 4,997,927.

McBride, L., McCollum, C., Davidson, S., Efcavitch, J.W., Andrus, A., and Lombardi, S. 1988. A new, reliable cartridge for the rapid purification of synthetic DNA. Biotechniques 6:362-367.

Mullah, B. and Andrus, A. 1996. Purification of 5'-O-trityl-On oligoribonucleotides. Investigation of phosphate migration during purification and detritylation. Nucleosides Nucleotides 15:419-430.

Contributed by Alex Andrus

PE Applied Biosystems

Foster City, California

Robert G. Kuimelis

Phylos, Inc.

Lexington, Massachusetts
Purification and Analysis of Synthetic Nucleic Acids and Components

$\mathbf{1 0 . 7 . 5}$ 